\title{
Fabricating Fiber Bragg gratings using phase modulated direct UV writing
}

\author{
C. Holmes, C. Sima, P.L. Mennea, L.G. Carpenter, J.C. Gates, P.G.R. Smith \\ Optoelectronics Research Centre, University of Southampton, Southampton, SO17 1BJ, UK.
}

\begin{abstract}
The authors present a direct UV writing approach to fabricate fiber Bragg gratings (FBGs) and gratings in photolithographic waveguides. The technique uses two coherent UV beams, which are focused to a small spot ( 7 $\mu$ m diameter) at the point at which they overlap. The resulting interference pattern at the foci consists of fringes which are used to define several grating planes per exposure, giving greater design flexibility and a significantly larger accessible spectral range compared to traditional approaches. The typical index contrast during grating fabrication is observed to be $4.7 \times 10^{-3}$, at writing speeds of $8 \mathrm{~mm} / \mathrm{min}$.
\end{abstract}

Keywords: Direct UV writing, Bragg gratings, FBGs, PLCs

\section{INTRODUCTION}

High quality fiber Bragg gratings (FBGs) with arbitrary phase and index profiles are increasingly desired in a wide range of applications including telecommunications, lasing systems and environmental monitoring. A simple approach to achieving such structures is to scan a UV beam over a long phase mask ${ }^{\mathbf{1}}$. Using this technique the flexibility in grating design is limited, as different structures can only be achieved through adjusting the hardware (i.e. using a different phase mask). A more flexible software based approach uses a dual beam interferometer alongside software controlled translation $^{2}$. This technique continually moves 100's of interference fringes as a fiber is translated. Other software based solutions include point-by-point direct femtosecond writing. This has allowed greater control of the grating design, as grating planes are individually defined ${ }^{3}$.

The technique presented in this paper effectively combines the advantages of these two approaches by using an interferometric arrangement that defines only a few $(<7)$ grating planes per exposure. Through doing this the design flexibility associated with point-by-point is combined with alignment and exposure averaging benefits of conventional dual beam interferometry. A comparison of this Direct UV Writing (DUW) technique compared to conventional dual beam interferometry and direct femtosecond writing has been constructed in table 1.

Table 1. A comparison between prior art dual beam interferometry; direct femtosecond writing and direct cw writing.

\begin{tabular}{|l|l|l|l|}
\hline \multicolumn{1}{|c|}{ Property } & Dual beam interferometry & Point-by-point direct writing & \multicolumn{1}{|c|}{ Direct (cw) UV writing } \\
\hline $\begin{array}{l}\text { Dynamic Range of } \\
\text { accessible periods }\end{array}$ & $\begin{array}{l}10 \text { 's nm (without adjusting } \\
\text { alignment) }\end{array}$ & In principle infinite & $\begin{array}{l}100 \text { 's nm (without adjusting } \\
\text { alignment) }\end{array}$ \\
\hline Alignment Complexity & $\begin{array}{l}\text { Large focused spot allows } \\
\text { larger alignment tolerances }\end{array}$ & $\begin{array}{l}\text { The focused spot is of the } \\
\text { dimension of a single period }\end{array}$ & $\begin{array}{l}\text { Spot size small but cw nature } \\
\text { permits alignment }\end{array}$ \\
\hline Design of discontinuity & $\begin{array}{l}\text { Made of 100's of grating } \\
\text { planes }\end{array}$ & Instantaneous & $\begin{array}{l}\text { Made over several }(<7) \text { grating } \\
\text { planes }\end{array}$ \\
\hline $\begin{array}{l}\text { Averaging of grating } \\
\text { planes }\end{array}$ & $\begin{array}{l}\text { Positional errors averaged over } \\
10 \text { 's of micrometers }\end{array}$ & $\begin{array}{l}\text { Each grating plane has } \\
\text { positional accuracy errors }\end{array}$ & $\begin{array}{l}\text { Positional errors averaged over } \\
\text { micrometers }\end{array}$ \\
\hline
\end{tabular}

This work reports direct UV writing of Bragg gratings into two waveguide geometries, optical fiber and photolithographic planar lightwave circuit (PLC). This work looks at the versatility of alignment and the properties of the gratings fabricated. Comment is also made upon a thermal effect observed during fabrication. 


\section{EXPERIMENTAL SETUP}

DUW takes full advantage of the photosensitive nature of germanosilicate glass. Typical refractive index contrasts are of the order of $5 \times 10^{-3}$, where the actual refractive index change made depends upon the photosensitivity of the substrate and total number of UV photons incident upon a point during the writing process. This is quantified in terms of a fluence parameter, F:

$$
\mathrm{F}=\frac{I_{U V} d}{v}
$$

Where $\mathrm{I}_{\mathrm{UV}}$ is average power intensity, $\mathrm{d}$ is the diameter of the spot in the core layer and $\mathrm{v}$ is the translation or writing velocity. The focused beams form a small writing spot of the order $\sim 7 \mu \mathrm{m}$ and typical writing fluences range from $5 \mathrm{kJcm}^{-2}$ to $30 \mathrm{kJcm}^{-2}$. This results in typical translation speeds (considering an incident UV laser power of $50 \mathrm{~mW}$ ) of $\sim 4 \mathrm{~mm} / \mathrm{min}$. Unlike similar femtosecond based direct writing techniques, this method does not work on the principle of non-linear effects ${ }^{3}$. Rather it is more comparable with UV exposed phase mask writing techniques, where an induced refractive index change is related to type I and type II processes occurring in a photosensitized silica core.

The phase modulated direct UV writing approach uses two focused coherent beams from a 244nm continuous wave UV laser. At their point of intersection the beams are focused to a $\sim 7 \mu \mathrm{m}$ diameter spot where they form an interference pattern, as illustrated in Figure 1. The arrangement is notably different to typical dual beam interferometric techniques [2], as the illustrated interference pattern has considerably fewer fringes.
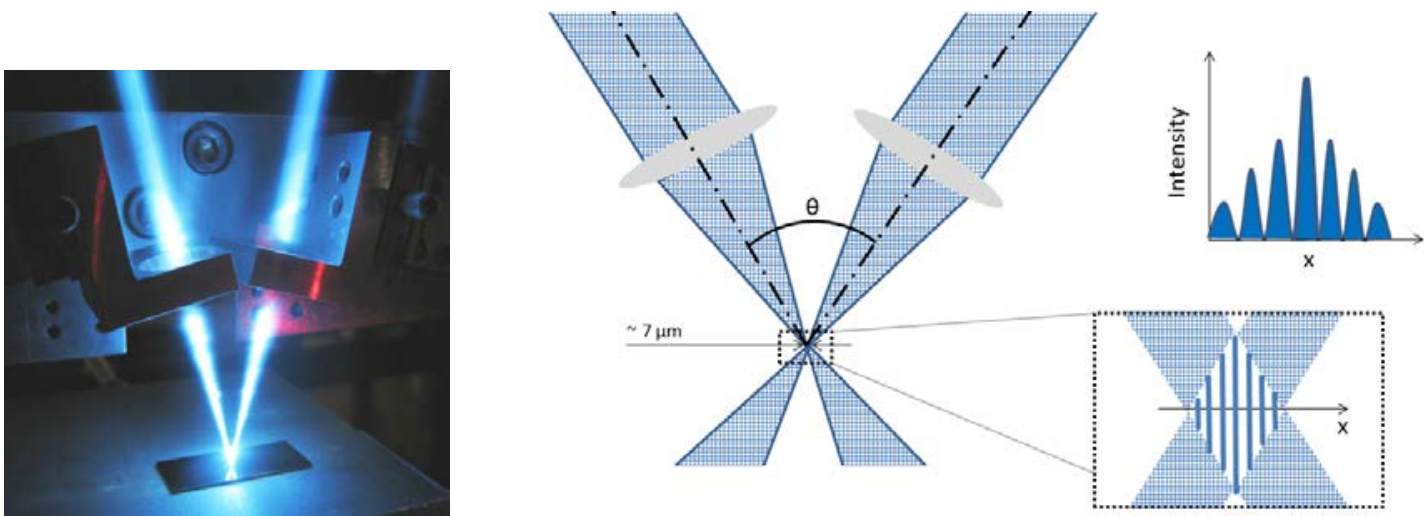

Figure 1. (left) a photograph of the direct UV writing arrangement (right) A conceptual image of the dual beam system; two coherent UV laser beams are overlapped at their focus forming several interference planes.

In order to construct Bragg gratings of millimeter length the interference pattern is traversed along the core of the waveguide. On translation the maxima and minima fringes are controlled through an induced path length variation of one arm. This has been achieved using an Electro-Optic Modulator (EOM), as illustrated in Figure 2.
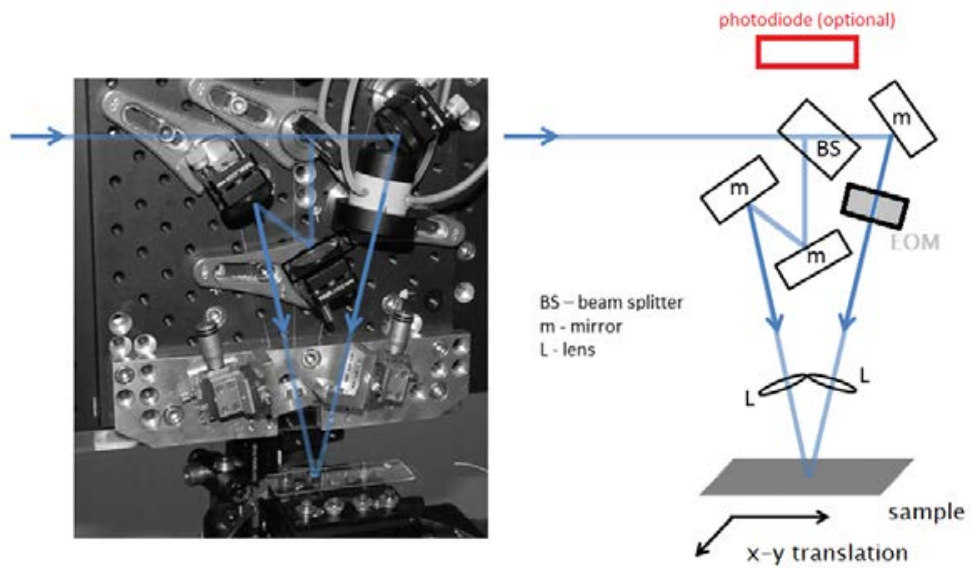

Figure 2. (left) a photograph of the direct UV writing interferometer (right) with a schematic of the optical arrangement. 
In the fabrication process the beam continually illuminates the sample as it is translated. As translation occurs the fringe pattern drifts such to maintain overlap to pre-exposures. This is achieved through controlling the phase of one path length, as illustrated in Figure 3. The drift in fringe pattern is set such that one complete cycle is equal to a translation $\Delta \mathrm{x}_{2}$ which is equal to the desired Bragg period. Detuning occurs if the translation $\Delta \mathrm{x}_{2}$ is either longer or shorter than the inherent fringe period of the cross-beam arrangement. A further description of this is given by Sima et $\mathrm{al}^{4}$.
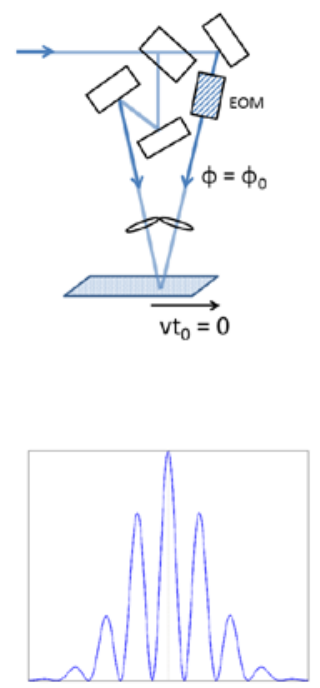
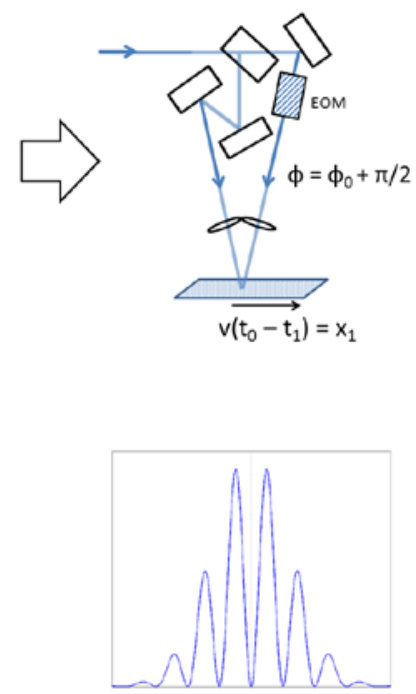
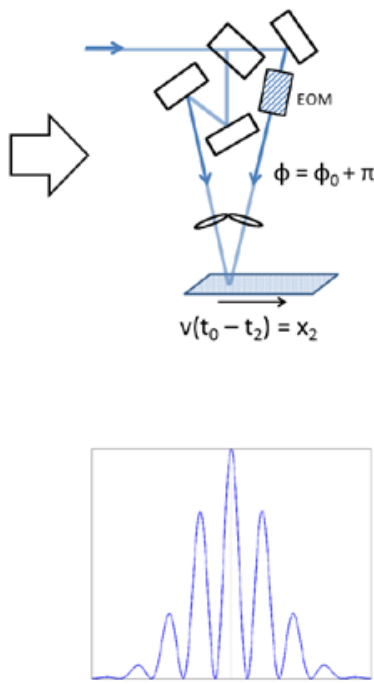

Figure 3. A schematic illustrating the optical set-up of the phase modulation system.

The technique of detuning provides a unique ability for a system optimized to write at 1550nm to access Bragg reflectors ranging from 1200 to $1900 \mathrm{~nm}$ without system realignment. As there are fewer fringes in the pattern a set crossing angle can define a large range of Bragg periods, compared to other interferometric approaches ${ }^{2}$. Detuning allows specific Bragg grating periods to be achieved, that do not necessarily match that of the initial interference fringe period.

Sample translation is achieved by using high precision air-bearing stages, with nanometer scale position resolution. The actuation of the EOM voltage undergoes positional synchronized firing with respect to translation of the stage system. The pulsed output is converted to a saw-tooth voltage signal via an integrator circuit which is then amplified to kV level and is then fed to the EOM, as illustrated in Figure 4.

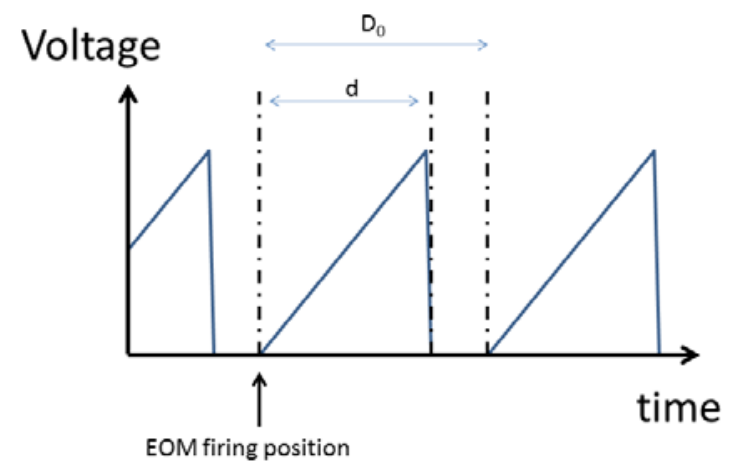

Figure 4 A schematic of the typical voltage signal applied to the EOM.

Within the applied voltage signal an effective exposure duty cycle can be applied. Referring to Figure 4, the duty cycle is the ratio of phase modulation provided by the EOM (d), compared to the total time of flight $\mathrm{D}_{0}$, which is equivalent to one complete Bragg period. Through control of duty cycle envelope functions can be applied to the grating, enabling a range of designs that can be achieved such as a Gaussian apodisation function.

The strength of this is technique is the use of a small spot, as it permits greater flexibility in grating design. However, this also brings additional alignment complexity. Alignment of the foci relative to the optical waveguide, be it an optical 
fiber or PLC, is achieved through use of a photodiode located at the top of the interferometer arrangement, as illustrated in Figure 2. Through monitoring the back reflected UV light on the diode, the position of a germanium doped core can be determined and from this alignment achieved. Figure 5 illustrates 10 linear scans made perpendicular to a silica-onsilicon PLC. These build up a 2-dimensional waveguide profile, which can be used for alignment. In the example the waveguide is germanium doped and so UV is absorbed prior and post reflection from the silica-silicon interface. This results in a dip in reflected power. A similar effect was also observed when scanning the core of a germanium doped fiber.
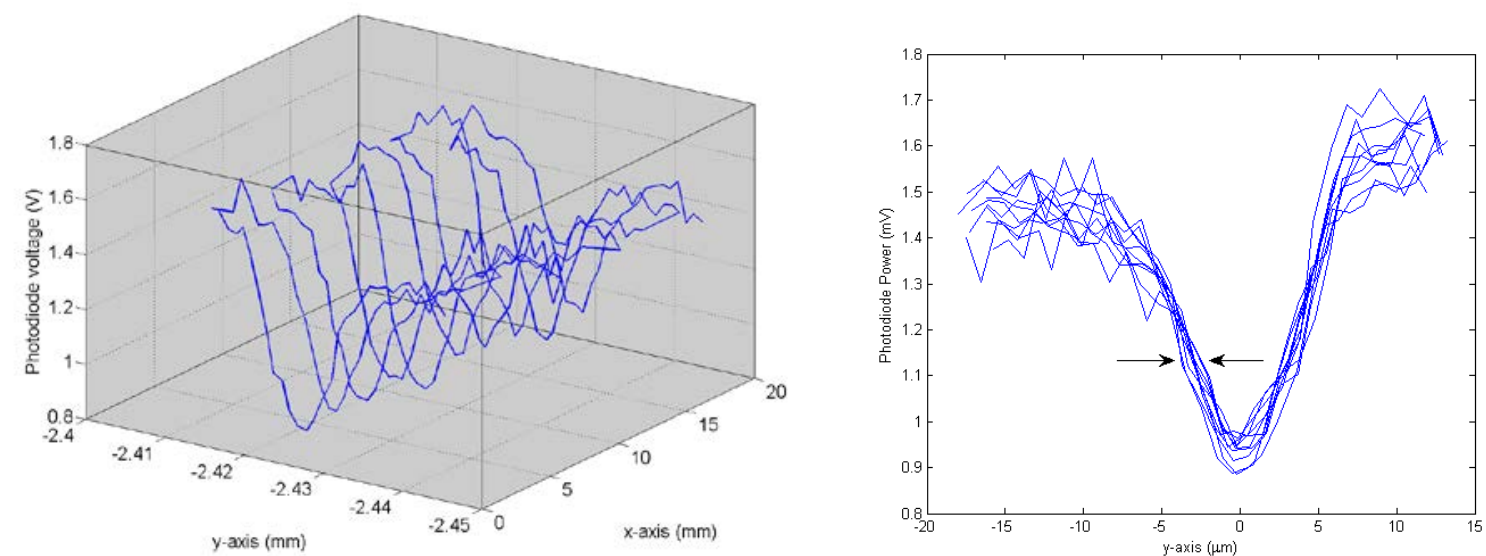

Figure 5 (a) An illustration of photodiode voltage as a function of laser position over a photolithographic waveguide. (b) Overlapping curves, with their statistical means overlapping (at $0 \mu \mathrm{m}$ ).

\section{GRATING DEMONSTRATION}

Figure 6 (a) illustrates duty cycle windowing to fabricate uniform (at the shorter wavelength) and Gaussian (at the longer wavelength) Bragg gratings in SMF-28. In this example the fiber is hydrogenated to enhance UV-photosensitivity and is written at a fluence of $5 \mathrm{kJcm}^{-2}$. The length of the uniform grating is $3 \mathrm{~mm}$ and the Gaussian grating is $15 \mathrm{~mm}$. The Gaussian apodised grating has been optimized to achieve a $3 \mathrm{~dB}$ bandwidth of $160 \mathrm{pm}$, with suppressed sidebands at the 20dB level.

Figure 6 (b) makes a theoretical fit to the uniform grating, using a transfer matrix method. From this it can be deduced that the induced index contrast was $4.7 \times 10^{-3}$, which is comparable to other FBG fabrication methods.

(a)

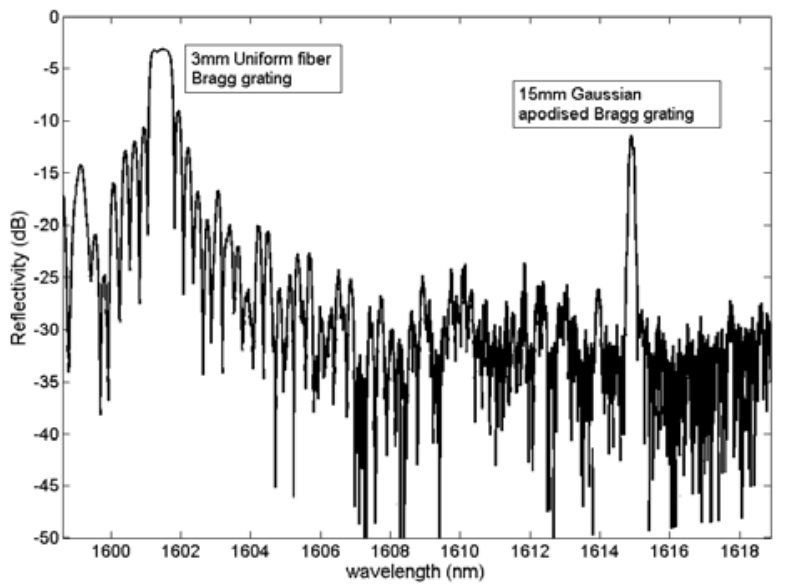

(b)

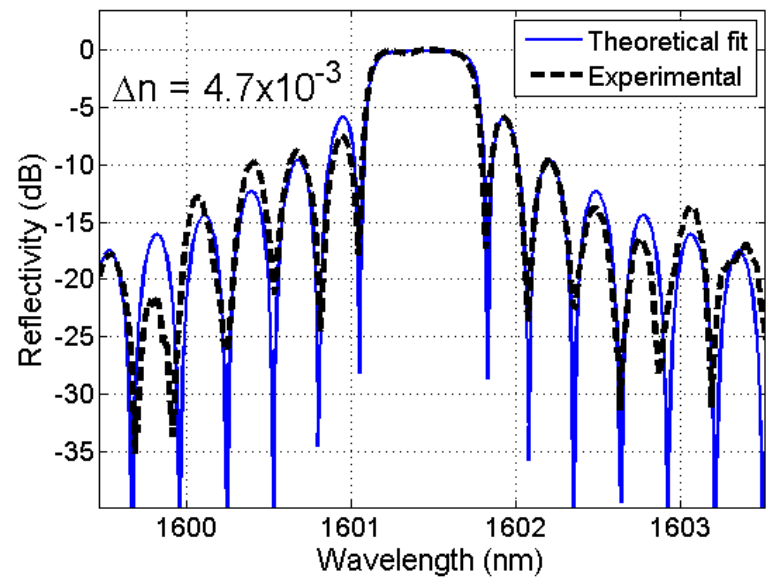

Figure 6 Two direct UV written Bragg gratings written into SMF-28. (a) the grating at shorter wavelength is 3mm long and has a uniform profile, the grating at longer wavelength has a Gaussian apodised profile. (b) fits to the experimental uniform grating with a transfer matrix method simulation, in order to approximate the index contrast. 
Through increasing fluence it is possible to achieve higher index contrasts. Additionally increasing fluence also affects birefringence. Figure 7 shows the fluence dependence on birefringence, for a photolithographic waveguide fabricated using flame hydrolysis deposition at the University of Southampton. It must be noted that this was all written without adjusting the alignment and thus Figure 7 (a) demonstrates part of the full range over which gratings can be accessed through the detuning method.

(a)

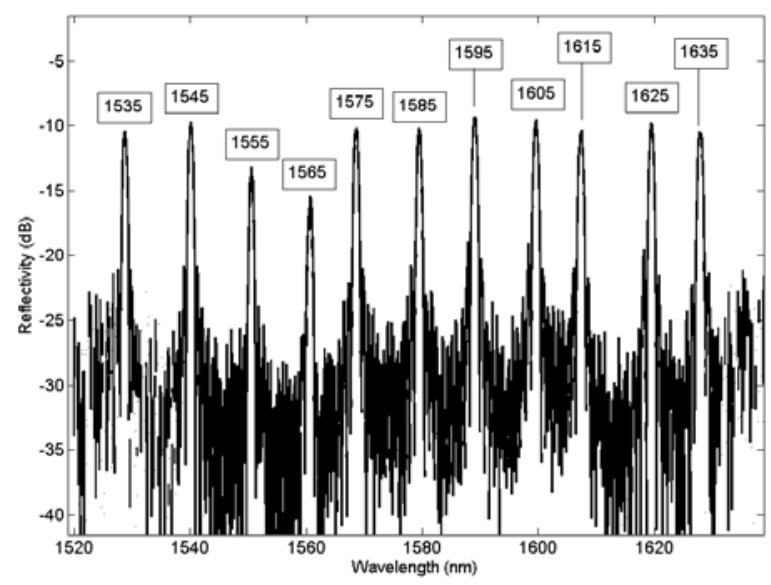

(b)

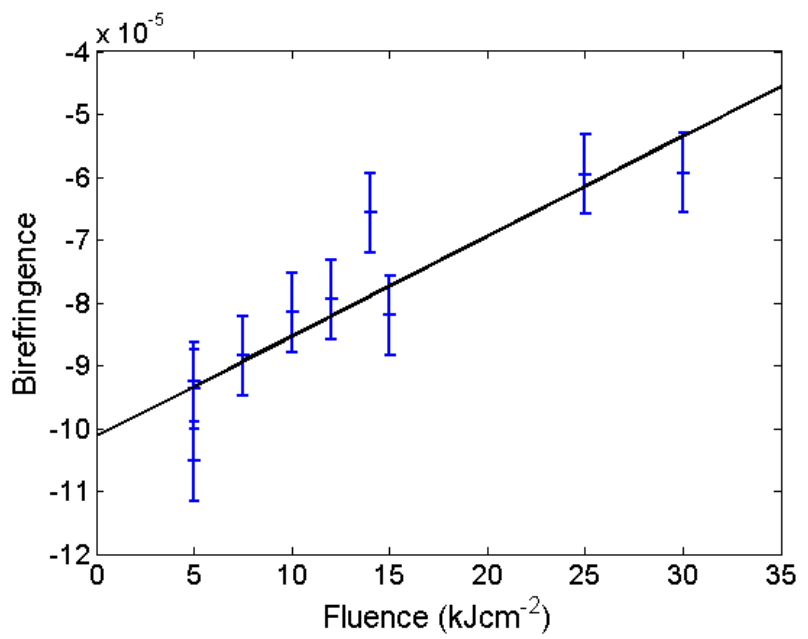

Figure 7 A series of $1 \mathrm{~mm}$ long Bragg gratings written into a $3.5 \mu \mathrm{mx} 3.5 \mu \mathrm{m}$ photolithographic waveguide, with a $1.5 \%$ index contrast. (a) The 11 successive gratings from short to long wavelengths have respective fluences of 5, 14, 25, 30, 5, 10, 7.5, 12, 3,15 and $5 \mathrm{kJcm}^{-2}$. (b) a plot showing the birefringence of the 11 gratings as a function of UV fluence.

The birefringence dependence on typical writing fluences is $\sim 1.6 \times 10^{-6} \mathrm{~kJ}^{-1} \mathrm{~cm}^{2}$.

During the UV writing of optical fiber thermal phenomena were observed. This was a direct result of the small writing spot and was a temporal effect that did not affect the quality of the final written grating.

\section{THERMAL EFFECT}

At the focus of the DUW cross-beam arrangement there is $\sim 50 \mathrm{~mW}$ of power at $244 \mathrm{~nm}$ wavelength focused to $\sim 7 \mu \mathrm{m}$ diameter spot, which corresponds to a radiative flux $\sim 1.3 \mathrm{GWm}^{-2}$. At this level significant heating occurs at the point of exposure. Experimental observation of this thermal effect is depicted in Video 1, for a SMF-28 fiber. In the video two gratings are consecutively written, the first at shorter and the second at longer wavelength. After the fabrication of the first grating a mechanical shutter is closed stopping UV exposure. This is subsequently reopened before the second grating is written.

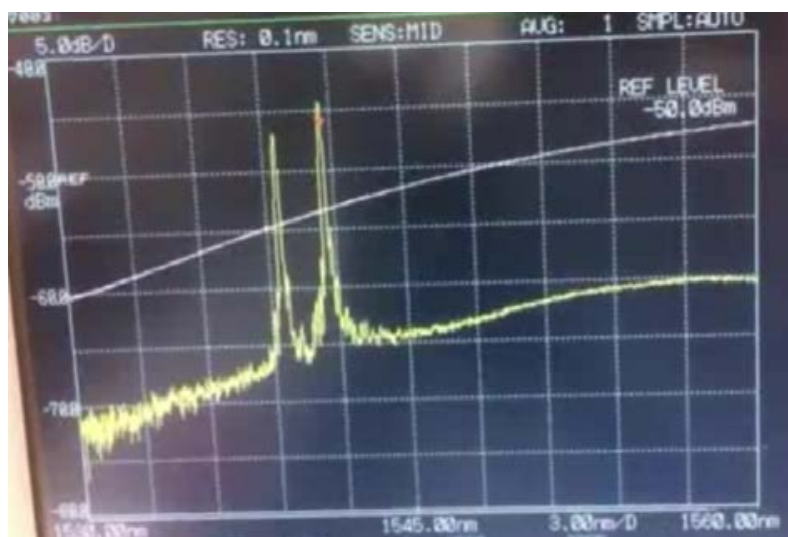

Video 1. A video showing the real time spectral evolution of a Gaussian apodised Bragg grating, indicating the thermal effect resulting from the high power density from the focused spot. http://dx.doi.org/doi.number.goes.here 
The grating can be observed to display a chirped response due to local heating as the grating is being written. It is also noted that when the shutter closes the chirp disappears and when it reopens it reappears. Writing of the second grating continues to effect the spectral response of the first grating (as well as the second) until both gratings are fully written.

Such thermal effects do not occur if the fiber is sufficiently thermally sunk, e.g. the use of water as a heat sink. It was noted that using this fabrication system there is no advantage, other than understanding spectral feedback during writing, which exists by using a heat sink medium. This was confirmed as gratings using both methods have a comparable set of spectral features after fabrication.

\section{CONCLUSIONS}

This work has demonstrated a DUW phase control technique for the fabrication of Bragg gratings in optical fiber and photolithographic waveguides. Through monitoring the back reflected UV light, it is possible to align the small (7 $\mu \mathrm{m})$ spot to the waveguide. Fringe index contrasts of $\sim 5 \times 10^{-3}$ have been observed in hydrogen loaded SMF-28, which is comparable to other techniques. A small birefringence dependence on fluence was measured to be $1.6 \times 10^{-6} \mathrm{~kJ}^{-1} \mathrm{~cm}^{2}$.

\section{REFERENCES}

[1] Martin, J. and Oulette, F., "Novel writing technique of long and highly reflective in-fiber gratings”, Electron Letters 34, 811-812 (1994).

[2] Petermann, I., Sahlgren, B., Helmfrid, S., Friberg, A.T. and Fonjallaz, P., "Fabrication of advanced fiber Bragg gratings by use of sequential writing with a continuous-wave ultraviolet laser source”, Applied Optics 41, 6, 1051-1056 (2002).

[3] Malo, B., Hill, K.O., Bilodeau, F., Johnson, D.C and Albert J., "Point-by-Point fabrication of Micro-Bragg gratings in photosensitive Fiber Using Single Excimer Pulse Refractive-Index Modification techniques”, Elect. Let. 40, 1170-1172 (2004).

[4] Sima, C., Gates, J.C., Rogers, H., Mennea, P.L., Holmes, C., Zervas, M., and Smith, P.G.R., "Ultra-wide detuning planar Bragg grating fabrication technique based on direct UV grating writing with electro-optic phase modulation,” Opt. Express 21, 15747-15754 (2013). 